\title{
New experimental setup for photoelectron spectroscopy on cluster anions
}

\author{
Chia-Yen Cha, G. Ganteför, and W. Eberhardt \\ IFF der KFA Jülich, Postfach 1913, D-5170 Jülich, Germany
}

\begin{abstract}
We describe a new experimental setup for photoelectron spectroscopy on mass selected clusters. The recently developed pulsed arc cluster ion source (PACIS) for metal and semiconductor clusters is used as an anion source. The design of the PACIS is optimized for maximum intensity of cluster ion production and a minimum internal temperature of the particles. A simple modification allows vaporization of liquid and low melting point metals. The produced anions are mass selected via an inline time-of-flight setup with the option of using a reflectron for increased mass resolution. Photoelectron spectra of the mass selected cluster anions are collected in a "magnetic bottle" type electron spectrometer. First results on copper clusters are presented.
\end{abstract}

\section{INTRODUCTION}

In experimental physics it is very often the development of a new experimental technique which will give rise to a quantum leap in the progress of the field. The area of metal and semiconductor cluster research ${ }^{1}$ is strongly dependent on the development of a source for these kinds of particles. With a few exceptions, the geometry and also the electronic structure of individual clusters are unknown at present. The limiting factors in the experimental determination of these properties are (i) the availability of intense cluster sources and (ii) a convenient and efficient way to produce a monosize mass selected beam of particles, if possible in the neutral ground state.

van der Waals clusters can be prepared in a molecular beam through cooling in an adiabatic expansion. ${ }^{2}$ Clusters of alkali atoms are produced by vaporization and condensation in a molecular beam after adiabatic expansion. ${ }^{3}$ However, most of the elements in the periodic system cannot be prepared as clusters with these techniques. A very intense source of clusters is the sputter source, ${ }^{4}$ but it produces only very small size clusters (up to 15 atoms). Therefore, in cluster science one of these above-mentioned quantum leaps was initiated by the development of the laser vaporization source (LVS). ${ }^{5}$ This source can be used for all solid materials. Only liquids like mercury and gallium cannot be vaporized with the LVS and the production of alkali clusters ${ }^{6}$ is difficult with the LVS.

The LVS can be combined with any pulsed spectroscopic technique. In the last five years photoelectron spectroscopy of cluster anions ${ }^{7,8}$ has become an increasingly important technique, allowing detailed studies of the electronic structure, while also providing some information about the geometry of these particles. ${ }^{9}$ The anions can easily be mass separated. The photodetachment process is a transition from the ground state of the anion into an eigenstate of the neutral cluster in the geometry of the anion. Therefore, these spectra contain information about the neutral states of the cluster, about which most of the theoretical data are available. The pulsed version of photodetachment spectroscopy, using a time-of-flight electron spectrometer, ${ }^{10}$ allows the use of pulsed lasers with higher photon energies (compared to $\mathrm{cw}$ techniques) and opens a deeper field of view into the valence-band states of a cluster.

In this paper we present a novel type of a pulsed cluster source which can be used for cluster production of materials in as wide a range as the LVS. The pulsed arc cluster ion source (PACIS) ${ }^{11}$ can produce neutral as well as negative and positive ions of metal and semiconductor clusters. The vaporization is done using a pulsed electric arc instead of a laser pulse. The advantages of the PACIS compared to the LVS are a higher intensity per pulse and a higher repetition rate than a LVS operating with a commercial excimer or Nd:YAG laser. All these advantages are achieved at a cost which is only a fraction of the cost of a LVS. Moreover, we also demonstrate the pulsed vaporization of liquid metals using the PACIS. In this report, the cluster beam properties of two versions of the PACIS (type I for solids and type II for liquids) are characterized by photoelectron spectroscopy of the cluster anions. With this new setup it is possible for the first time to study gallium cluster anions ${ }^{12}$ with photoelectron spectroscopy at moderate resolution $(\sim 30 \mathrm{meV})$.

Ultraviolet photoelectron spectroscopy (UPS) of cluster anions can be carried out using conventional electrostatic analyzers. ${ }^{7,13}$ These types of spectrometers typically operate at a resolution of $10 \mathrm{meV}$, but suffer from low counting rates because of the small acceptance angle of the spectrometer. The collection efficiency for the photoelectrons can be increased by a factor of about $10^{3}$ using a magnetic bottle type time-of-flight (TOF) analyzer. ${ }^{10}$ While a magnetic bottle spectrometer yields a resolution of 10-20 meV for neutral, thermal gas phase species, for anions this technique suffers from a relatively low resolution $(100 \mathrm{meV})$ caused by Doppler broadening due to the high kinetic energy of the anions $(>100 \mathrm{eV})$. In the experimental setup which is described here, the anions are decelerated shortly before the detaching laser hits the anion bunch. This drastically reduces the Doppler broadening in the electron spectra and improves the resolution without a loss in intensity. Indeed, the intensity is enhanced due to the spatial compression of the anion bunch. A resolution of $30 \mathrm{meV}$ is achieved.

The experimental capabilities can be further enhanced through the use of zero electron kinetic energy spectros- 


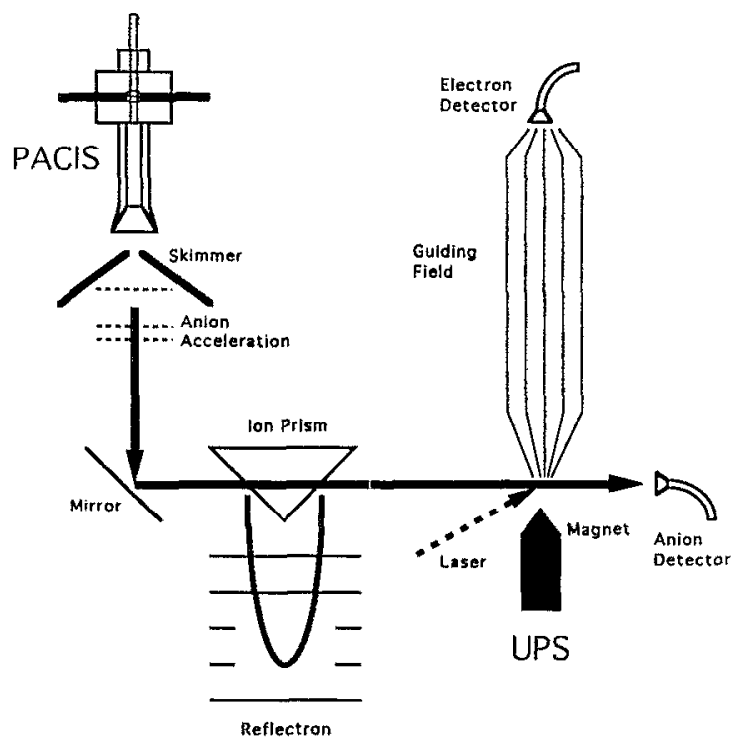

FIG. 1. Schematic of the experimental setup. Positively and negatively charged metal clusters are produced by the PACIS. After cooling in a supersonic expansion they pass the skimmer and are accelerated in the source region of a Wiley McLaren time-of-flight mass spectrometer. The anion beam is deflected by $90^{\circ}$ of the neutral beam axis. The anion beam can be either directly passed through to the ultraviolet photoelectron spectrometer (UPS) or can be sent through a reflectron prior to entering the electron spectrometer. Electrons are detached from a single bunch of anions by a UV laser pulse. The kinetic energy of the photoelectrons is measured in a magnetic bottle time-of-flight spectrometer.

copy (ZEKES). ${ }^{14}$ The extremely high resolution $(<1$ $\mathrm{meV}$ ) enables the determination of vibrational substates in the observed transitions and eigenstates, which is especially interesting for ground-state transitions. However, UPS data are necessary as a guide for the application of such a time-consuming high-resolution technique as ZEKES.

\section{THE EXPERIMENTAL SETUP}

A schematic of the apparatus is shown in Fig. 1. It consists of the PACIS, the time-of-flight mass spectrometer, and the UPS spectrometer. In the following, each part of the apparatus will be discussed in detail.

\section{A. The pulsed arc cluster ion source}

The PACIS consists of three main parts: the source body, the power supply, and the vacuum system.

\section{The source body}

Two variations of the scurce body have been constructed: Type I is used for solids and semiconductors with good or moderate electrical conductivity [Fig. 2(a)] and type II is used for liquid metals and alkali clusters [Fig. 2(b)].

In the type I [Fig. 2(a)] source the arc burns within the center of a small ceramic cube $(\sim 2 \times 2 \times 2 \mathrm{~cm})$. This cube has two channels perpendicularly intersecting each other in the center. The electrodes (diameter $d=5 \mathrm{~mm}$ ) are inserted from opposite sides into the larger bore. They face each other at the center of the cube leaving a gap of about $1 \mathrm{~mm}$. A carrier gas pulse (usually helium), pro-

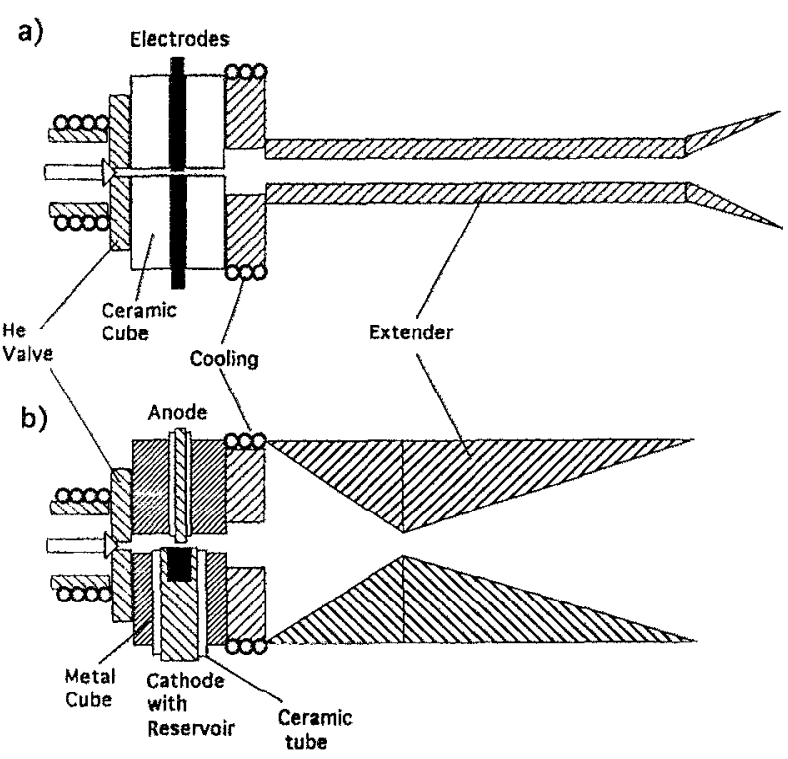

FIG. 2. Schematic view of the two versions of the PACIS used in this experiment: (a) type I for conducting solids and (b) type II for liquids and alkali metals. The two extenders shown in the figure can be combined with each type. The optimum design of the extenders depends on the material and the charge state of the ions to be produced.

duced by a pulsed valve (General Valve solenoid valve), flows through the second channel and the gap between the electrodes. This channel has a rectangular shape $(1 \times 5$ $\mathrm{mm}^{2}$ ) to match the gas stream to the geometry of the gap between the electrodes. Thus, through the whole gap a strong gas flow is ensured. The carrier gas leaves the ceramic cube on the opposite side streaming into the extender.

Simultaneously with the gas pulse the electric are is ignited. For about 3-20 $\mu$ s a current of $5000 \mathrm{~A}$ flows between the two electrodes. Only the cathode is eroded. We assume the dominant mechanism for erosion is ion sputtering due to the relatively high electric field in the gap ( 10 $\mathrm{kV} / \mathrm{cm}$ ). The metal vapor and the helium mixes within the discharge and the resulting plasma is flushed into the extender. Depending on the kind of clusters to be produced, the design of the gas channel is more or less crucial. A strong cluster signal is found for $\mathrm{Al}, \mathrm{Ga}, \mathrm{Mo}$, and $\mathrm{C}$, nearly independent of the details of design of the source body. However, it was difficult to obtain a stable and intense output for copper clusters. Copper seems to be eroded to a much smaller extent by the electric discharge and thus any losses of metal vapor at corners and on surfaces in the channel and the extender are detrimental.

In the source a competition between the electric field force and the pressure of the carrier gas stream takes place. Only if a high gas pressure and flow are achieved within the gap, sufficient amounts of material are transported into the extender. In a version of the source body having a much larger diameter of the gas channel, which results in a lower flow of gas through the gap, a collar of deposited copper was found around the cathode. This anisotropy of deposition of material is caused by the presence of the electric field which accelerates the positive ions into the 
direction of the cathode. If the eroded material would behave like neutral atomic vapor, the walls around the gap would be homogeneously covered. Since this is not the case, we conclude that most of the eroded metal is positively charged. The negative ions and neutrals obviously must be generated by a charge exchange or neutralization mechanism, which probably takes place during the cooling in the extender.

One important difference between the PACIS and the LVS is the large amount of energy deposited into the carrier gas. The helium traveling through the arc is converted into a hot plasma. Thus, more effort has to be taken to cool down the mixed helium/metal plasma. If a high output of positive or negative ions is desired, a compromise has to be found between cooling and neutralization. Several different shapes of extenders were compared and depending on the material and the charge state different designs wcre found to give the optimum performance. The two examples shown in Figs. 2(a) and 2(b) give reasonable results in most cases. The extender must be water or liquid $\mathrm{N}_{2}$ cooled.

In type II of the PACIS [Fig. 2(b)] the arc burns inside a molybdenum cube. The electrodes are insulated against the grounded metal cube by ceramic tubes. The arc burns in a small chamber in the cube with a minimum distance of $1 \mathrm{~mm}$ to the walls. The metal walls do not disturb the electric arc. The reason is probably that the pulsed power supply is ground free and any contact of the arc with the walls is the only contact of the discharge circuit with ground potential. The electrodes are arranged vertically. The upper electrode (anode), which does not erode, is a rod of molybdenum $(d=2 \mathrm{~mm})$. The lower electrode (cathode) is a rod of molybdenum $(d=8 \mathrm{~mm})$ with a $7 \mathrm{~mm}$ hole at the top. The hole serves as a reservoir for the target material. In this design the gas flow through the electrode gap is smaller (compared to type I) because of the larger distance of the walls of the cube to the arc. A significant amount of gas flows only around the two electrodes. This lowers the yield of vaporized metal, but on the other hand, the low melting point materials used in this source produce sufficient amounts of metal vapor, even at low discharge currents. In the mass spectra we found no traces of molybdenum, indicating no or very little erosion of the electrodes or the housing.

Normal operation requires daily maintenance. The outlet channel of the ceramic cube (type I) tends to clog and the cathode must be realigned to compensate for the erosion of the material (about $1 \mathrm{~mm} / \mathrm{day}$ ). For gallium (type II) stable operation is possible for several days.

\section{The power supply}

The high current necessary for the PACIS is delivered by a fast discharge of a capacitor. The capacitor is loaded up to $960 \mathrm{~V}$ between each ignition. The capacitor is connected to the two electrodes via a hockey puck thyristor. The current through the thyristor is limited to a maximum value of $5000 \mathrm{~A}$ by a resistor. The size of the capacitor can be varied depending on the material to be vaporized. For liquids a few microfarads are sufficient while for copper we use the maximum installed capacity of $80 \mu \mathrm{F}$. Typical time constants of the discharge are $2-20 \mu \mathrm{s}$. The thyristor blocks when the discharge current drops below a certain value. Then the capacitor is loaded up again through a second thyristor which blocks when the loading current drops close to zero. Timing electronic ensures the correct triggering of the two thyristors.

For lifetime reasons the high-power thyristor is overdimensioned (20000 A maximum pulse current, $2500 \mathrm{~V}$ maximum voltage). If the PACIS does not ignite, which might occur in case the electrode gap is too large, the capacitor has to be discharged through a parallel resistor. Without this resistor the thyristor can be destroyed by delayed ignitions. The time constants for charging and discharging the capacitors limit the maximum frequency of the PACIS. With a maximum peak loading current of 20 $A$, the repetition frequency is limited to about $20 \mathrm{~Hz}$ depending on the size of the capacitor. With a more powerful dc supply frequencies of several hundred $\mathrm{Hz}$ should be possible. However, in the setup described here the overall limitation is given by the pumping speed (see below) and the repetition rate of the photodetaching laser $(10 \mathrm{~Hz})$.

The dc power supply for loading the capacitor consists of three transformers with $220 \mathrm{~V}$ ac output. These are high voltage isolated and high frequency shielded. This ensures a very low hf disturbance caused by the discharge. The ac outputs are rectified and the output serially coupled to about $960 \mathrm{~V}$ maximum output. This de power supply delivers a peak loading current of $20 \mathrm{~A}$.

\section{The vacuum system}

The vacuum system is very similar to a standard setup for LVS. ${ }^{5}$ The gas load is higher because a relatively high gas flow through the gap of the electrodes is necessary. The PACIS body is mounted inside a 150 CF cross, pumped by a $2200 \ell / \mathrm{s}$ oil diffusion pump. This relatively small pumping speed limits the repetition rate of the source to about 20 Hz. While the source is running the pressure is about 5 $\times 10^{-4}$ mbar. The skimmer $(d=5 \mathrm{~mm})$ is mounted between the source chamber and the first differential pumping stage. The differential pumping stage is connected to a $2200 \ell / \mathrm{s}$ oil diffusion pump maintaining a pressure of 3 $\times 10^{-5}$ mbar during operation. The second differential pumping stage is pumped by a $400 \mathrm{\ell} / \mathrm{s}$ turbopump (pressure $=1 \times 10^{-6} \mathrm{mbar}$ ), while the UPS chamber is kept at about $1 \times 10^{-9}$ mbar by a $300 \mathrm{l} / \mathrm{s}$ second turbopump. The UPS chamber is bakeable and a liquid $\mathbf{N}_{2}$ trap can be connected to the system to improve the vacuum.

\section{B. The time-of-flight mass spectrometer}

As shown in Fig. 1 the ions and anions produced by the PACIS are accelerated by a pulsed electric field inline with the supersonic jet. This ion extraction is configured as a two-stage Wiley McLaren time-of-flight mass spectrometer. $^{15}$ The ions are deflected by $90^{\circ}$ by an electrostatic mirror to separate the ion beam and the neutral beam. The source produces an intense UV light flash, which creates photoelectrons on all surfaces downstream. Therefore, the 
UPS beam axis is orthogonal to the source beam axis. Closely behind the ion mirror an electrostatic biprism is installed. If the biprism is grounded the beam passes unreflected. If it is switched on, the ion beam is deflected into a reflectron. This reflectron returns the ion beam back to the other side of the biprism and the ions are deflected back onto the axis of the UPS spectrometer.

The electric fields of the reflectron are inhomogeneous to achieve a focusing effect. With simple homogeneous fields the profile of the reflected beam becomes too large to focus it into the small apertures of the UPS spectrometer. Using the reflectron causes a gain in mass resolution of about a factor of 5 at a simultaneous loss in intensity by about an order of magnitude. The reflectron will be used for studies of adsorbates on clusters, where a higher mass resolution is necessary.

The resolution of the inline Wiley McLaren time-offlight mass spectrometer depends strongly on the beam intensity. At high ion intensities space-charge effects in the starting area cause a line broadening. At normal conditions a mass resolution of $m / \Delta m=50$ is achieved. With the additional usc of the reflectron the resolution is enhanced to $m / \Delta m=250$.

\section{The ultraviolet photoelectron spectrometer}

For the acquisition of photoelectron spectra the source is optimized for the production of anions and the mass spectrometer switched to negative particle acceleration. The anion bunches of different masses are selected by their time of arrival at the source region of the photoelectron spectrometer. The electrons are detached from a certain cluster anion bunch by an excimer laser pulse (photon energy $3.5-7.9 \mathrm{eV}$, photon flux $100 \mathrm{~mJ} / \mathrm{cm}^{2}$, pulse length 5-20 ns). Adjusting the time delay between the cluster source (ion extraction field) and the photodetaching laser the photoelectron spectra can be recorded for individual mass cluster anions.

The electrons are guided by magnetic fields from the interaction region between the laser to a channeltron through a drift tube of a length of $1.8 \mathrm{~m}$. The magnetic field has the shape of a bottle ("magnetic bottle" type electron spectrometer). ${ }^{16}$ The electrons are created in a strong inhomogeneous field $(1500 \mathrm{G})$, which decreases at a distance of a few centimeters to a constant value of $2 \mathrm{G}$ in the drift tube. While passing through the gradient region the velocity vectors of the electrons are aligned parallel to the axis of the drift tube. Because of this alignment, the time between the laser pulse and the arrival of the electrons at the channeltron detector directly yields the kinetic energy. The difference between the photon energy and the kinetic energy is the binding energy.

For low kinetic energy electrons as in our case, a large fraction of the emitted electrons can be guided towards the detector by the magnetic field. In our case, the electrons are created within the inhomogeneous part of the magnetic field. Electrons with sufficient low energy, which have a velocity component in the direction of increasing magnetic field, turn around and are also guided towards the detector.

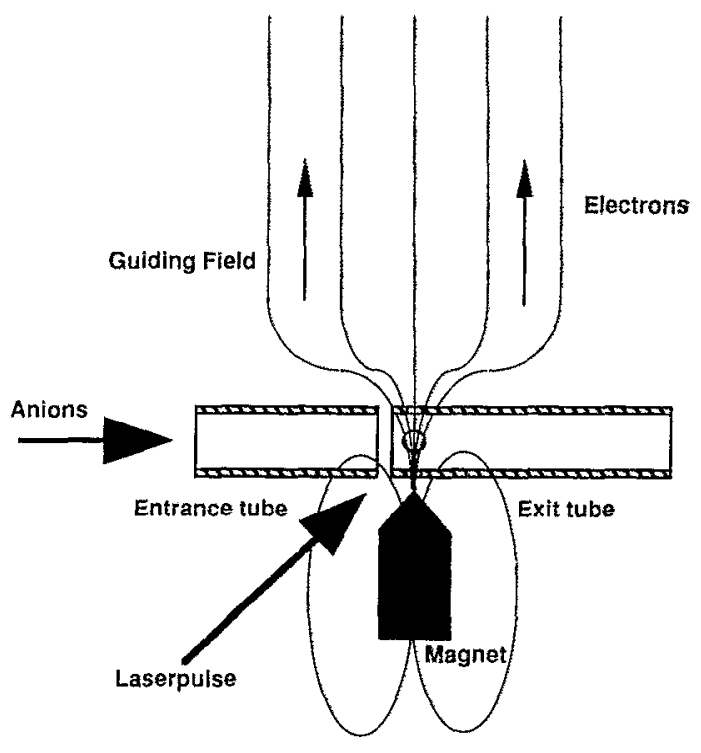

FIG. 3. Schematic view of the interaction area of the ultraviolet photoelectron spectrometer. While the anions pass the entrance tube the potential of the tube is switched from ground potential to a positive value corresponding to the beam energy. In the gap between the two tubes the anions are decelerated and stopped inside the second tube above the tip of the permanent magnet. A laser pulse detaches electrons from the anions. The electrons are guided by the magnetic field into the direction of the decreasing gradient. The electric field used to stop the anions has to be switched off before the laser pulse.

The divergent magnetic field acts as a magnetic mirror. Thus almost $100 \%$ of the detached electrons are detected.

The strong, highly inhomogeneous field is generated by a small cylindrical permanent magnet with a soft iron tip to enhance the field in the center of the interaction region where the two beams intersect. The guiding field is generated by a coil outside the vacuum vessel. The axis of the magnetic bottle spectrometer is aligned parallel to the earth's magnetic field. No additional magnetic shielding is used. Local deviations of the magnetic field are corrected by additional coils. All surfaces near the electron beam are coated with graphite to ensure a homogeneous work function.

Since the electrons reach the detector nearly independent of the angle of emission in the photodetachment process, Doppler broadening is caused by the velocity of the anions in the source region. The center of mass velocity of the anions must be added to the velocity vectors of the electrons. At a beam energy of $300 \mathrm{eV}$ the Doppler broadening of electrons of $1 \mathrm{eV}$ kinetic energy emitted from an anion of mass 100 amu results in an apparent energy spread of $160 \mathrm{meV}$. This effect becomes even more dramatic for lighter clusters like Al or $\mathrm{Li}$. Since it is inefficient to operate the ion extractor and mass selector at lower energies, we have developed a pulsed field technique to stop a selected cluster anion bunch at the interaction region of the electron spectrometer.

The anions enter the source region of the electron spectrometer through a 5-cm-long metal tube with a diameter of $4 \mathrm{~mm}$ (Fig. 3). While the selected bunch is passing through the tube, the potential of the tube is raised from 
zero (=ground) to a voltage corresponding to the ion beam energy (e.g., $+300 \mathrm{eV}$ ). The tube ends about $4 \mathrm{~mm}$ in front of the interaction region. After passing a gap of 1 $\mathrm{mm}$, the bunch enters a similar tube, which is always kept at ground potential. The two tubes form an electrostatic lens for the anion beam. The anions are decelerated due to the difference in potential and, depending on their exact kinetic energy, turn around at a certain distance within the second tube. By adjusting the voltage applied to the entrance tube the turnaround point can be positioned at the center of the interaction region, which is located at about 3 $\mathrm{mm}$ downstream within the second tube. Due to the spatial focusing effect of the Wiley McLaren mass spectrometer the anions exhibit a certain spread in kinetic energy. Thus stopping is only achieved for a mean energy. The two tubes with the gap define an electrostatic lens with a very short focal length. At certain conditions the turnaround point and the focus are identical. Thus a spatial compression of the bunch in all three dimensions increases the target density at the interaction region of the electron spectrometer.

The fast switching of the first tube to a potential very close to zero $(<20 \mathrm{meV})$ is a technical challenge. About $0.3 \mu$ s before the laser pulse hits, any electric field close to the source region has to be switched off. We have solved this problem by an overshoot technique. In our electronic circuit the voltage drops below zero $(-40 \mathrm{~V})$, but a fast Schottky diode blocks the connection to the tube which is kept at ground potential by a resistor.

The energy resolution obtained with this spectrometer is $25 \mathrm{meV}$ at best and $30 \mathrm{meV}$ in the average nearly independent of the cluster mass. The typical acquisition time for one of the spectra shown below was 5-10 min.

\section{RESULTS AND DISCUSSION}

\section{A. Performance of the PACIS}

The output of the PACIS is monitored with the timeof-flight mass spectrometer. Without an extender the source produces a very high intensity of helium and metal positive ions. No anions are observed in this mode. After installation of an extender (Fig. 2), the helium ion peak disappears and negatively as well as positively charged clusters are generated. Therefore in practice, the optimum extender design depends on the material and desired charge state of the clusters. Figure 4 displays mass spectra of positive copper clusters and negative gallium clusters, obtained with the two different types of the PACIS. The spectra do not display the correct relative intensities because of strong saturation effects of the channelplate detector. Although we did not concentrate on increasing the performance for generation of positive ions, the PACIS seems to produce higher intensities of positively than of negatively charged clusters.

For several reasons discussed below it is difficult to obtain accurate values for the ion intensity. The ion detector (see Fig. 1) located behind the UPS can be replaced by a Faraday cup. The total mass integrated current is $0.1 \mathrm{nA}$ at $10 \mathrm{~Hz}$ for $\mathrm{Cu}_{n}^{+}$clusters ( $n=1-20$ atoms). Thus, each bunch of ions of a defined mass contains about $10^{6}$ parti-

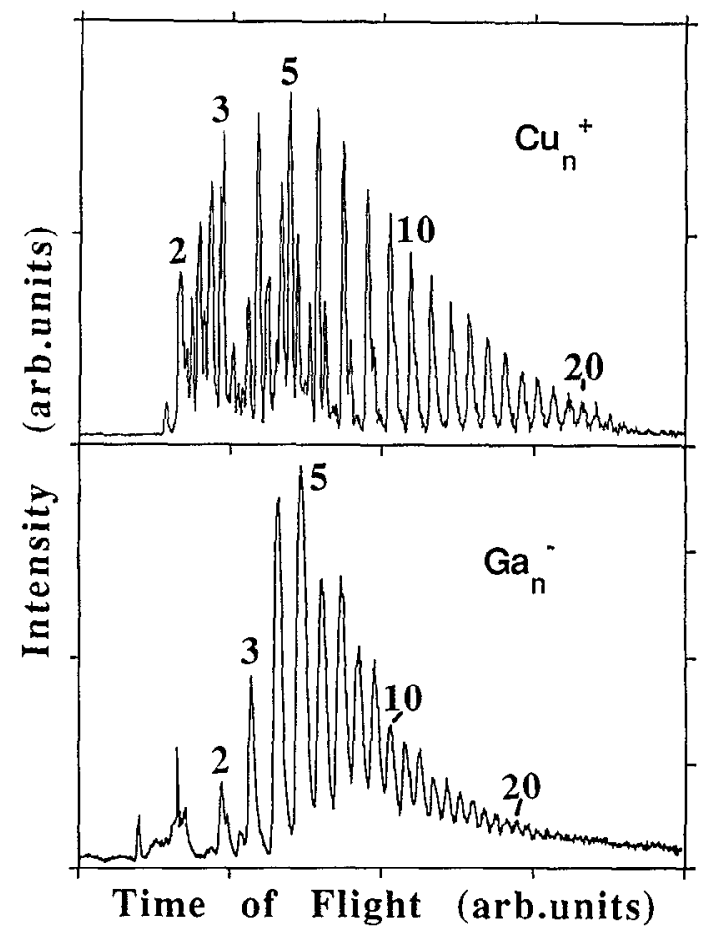

FIG. 4. Typical mass spectra of $\mathrm{Cu}_{n}^{+}$(type I PACIS) and $\mathrm{Ga}_{n}^{-}$(type II PACIS) ions generated by the PACIS. The two spectra were recorded at different acceleration voltages and are plotted with different zero offset. The spectrum of $\mathrm{Cu}_{n}^{+}$clusters is a sum of spectra taken over a limited size range and does not reproduce the true intensity ratios. The copper spectrum is contaminated with oxygen. The mass resolution is limited by space-charge effects in the source region of the time-of-flight spectrometer.

cles. The total positive current entering the first differential pumping stage can be measured by placing a negatively biased Faraday cup closely behind the skimmer opening. An integral beam current of about $100 \mathrm{nA}$ at $10 \mathrm{~Hz}$ is measured at this point.

The loss of intensity downstream can be explained by two effects:

(1) Only ions inside the source region of the Wiley McLaren time-of-flight spectrometer are accelerated by the pulsed field. The length of this area is about $0.05 \mathrm{~m}$, thus much smaller than the length of the ion plume produced by the source (about $0.5 \mathrm{~m}$ ). Thus only $10 \%$ of the ions are accelerated.

(2) The particles travel in a supersonic beam. However, since the particles are charged, electrostatic interactions cause a fast blowing up of the beam as soon as the particles enter the region of low pressure behind the skimmer. As a result, part of the ions hit the walls before being accelerated. In addition, the beam becomes highly divergent, which causes losses downstream where it is focused through several small apertures.

\section{B. Test of the electron spectrometer}

The "magnetic bottle" electron spectrometer was tested and calibrated using $\mathrm{Cu}_{n}^{-}$clusters. Figure 5 displays a comparison between photoelectron spectra obtained in Lineberger's group ${ }^{17}$ (top panels) and data from our new 


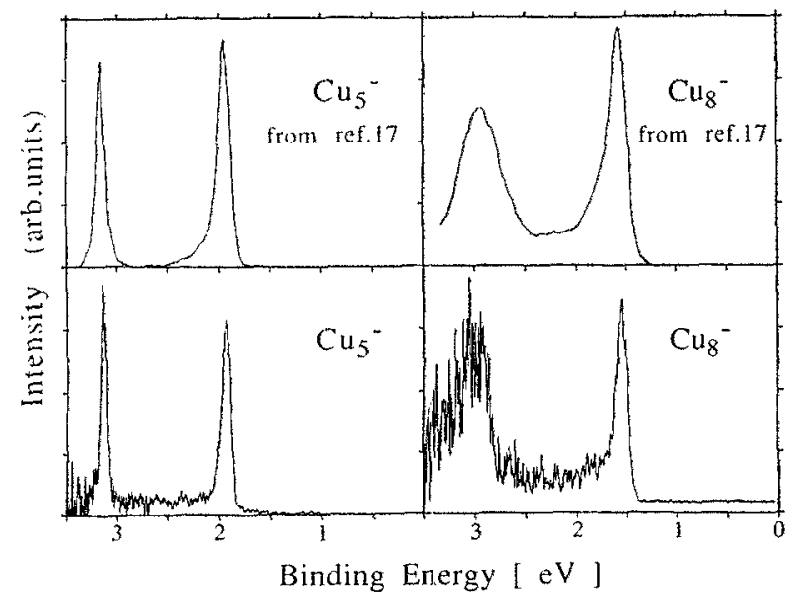

FIG. 5. Comparison of UPS spectra of $\mathrm{Cu}_{5}^{-}$and $\mathrm{Cu}_{8}^{-}$(lower panels) with data from the literature (See Ref. 17) (upper panels). In all spectra the observed linewidths are larger than the experimental resolution. The spectra taken from the literature are only a schematic copy of the original data.

electron spectrometer (bottom panels). The photon energy in both experiments was about $3.5 \mathrm{eV}$. The observed photoelectron linewidths in the four spectra are larger than the experimental resolution (Lineberger: $5-8 \mathrm{meV}$, here: $25-$ $30 \mathrm{meV}$ ). In the case of $\mathrm{Cu}_{5}^{-}$the two spectra are nearly identical. For $\mathrm{Cu}_{8}^{-}$, our spectrum exhibits a slightly smaller linewidth and steeper slopes, especially for the feature at $3 \mathrm{eV}$ binding energy. This feature also seems to exhibit a double structure which is unresolved in the earlier spectra. These data will be discussed in detail elsewhere. ${ }^{18}$

The narrower linewidths can be explained by a lower vibrational temperature of the anions produced by the PACIS. The temperature of the anions produced by the source used in Ref. 17 has been estimated from the vibrational temperature of the dimers to about $350 \mathrm{~K}$. Thus, the vibrational temperature of the anions produced by the PACIS is lower than $350 \mathrm{~K}$. Until now, we were not successful in resolving vibrational substructure in photodetachment, which should be observable for the $\mathrm{Cu}_{2}^{-}$dimer. The kinetic energy of electrons detached from $\mathrm{Cu}_{2}^{-}$is about $2.9 \mathrm{eV}$. At this relatively high energy, the resolution of our spectrometer is not sufficient to resolve these vibrations, which have a spacing of $30 \mathrm{meV}$.

\section{ACKNOWLEDGMENTS}

We thank S. Krummacher and P. Bechthold for their support during the buildup phase of the experiment. Special thanks also to $\mathrm{K}$. H. Meiwes-Broer and the group in Bielefeld for their cooperation.

${ }^{1}$ See, for example, Proceedings of the Fifth International Meeting On Small Particles and Inorganic Clusters, Konstanz, Federal Republic of Germany, September 1990, edited by O. Echt and E. Recknagel, $Z$. Phys. D $19+20(1991)$.

${ }^{2}$ O. Echt, K. Sattler, and E. Recknagel, Phys. Rev. Lett. 47, 1121 (1981); T. D. Märk, Int. J. Mass Spectrom. Ion Proc. 79, 1 (1987); G. Ganteför, G. Bröker, E. Hollub-Krappe, and A. Ding, I. Chem. Phys. 91, 7972 (1989); J. Stapelfeldt, J. Wörmer, and T. Möller, Phys. Rev, Lett. 62, 98 (1989).

${ }^{3}$ C. Bréchignac, Ph. Cahuzac, and J. Ph. Roux, J. Chem. Phys. 87, 229 (1987); W. D. Knight, K. Clemenger, W. A. de Heer, W. Saunders, M. Y. Chou, and M. L. Cohen, Phys. Rev. Lett. 52, 2141 (1984); C. R. C Wang, S. Pollack, D. Cameron, and M. M. Kappes, J. Chem. Phys. 93, 3787 (1990); T. P. Martin, T. Bergmann, H. Göhlich, and T. Lange, Chem. Phys. Lett. 172, 209 (1990).

${ }^{4}$ P. Fayet and L. Wöste, Z. Phys. D 3, 177 (1986); W. Begemann, K. H. Meiwes-Broer, and H. O. Lutz, Phys. Rev. Lett. 56, 2248 (1986); W. Eberhardt, P. Fayet, D. M. Cox, Z. Fu, A. Kaldor, R. Sherwood, and D. Sondericker, ibid. 64, 780 (1990).

${ }^{5}$ V. E. Bondebey and J. H. English, J. Chem. Phys. 74, 6978 (1981); T. G. Dietz, M. A. Duncan, D. E. Powers, and R. E. Smalley, ibid. 74, 6511 (1981).

${ }^{6}$ E. C. Honea, M. L. Homer, J. L. Persson, and R. L. Whetten, Chem. Phys. Lett. 171, 147 (1990).

${ }^{7}$ D. G. Leopold, J. H. Ho, and W. C. Lineberger, J. Chem. Phys. 86, 1715 (1987)

${ }^{8}$ O. Cheshnovsky, K. J. Taylor, J. Conceiao, and R. E. Smalley, Phys. Rev. Lett. 64, 1785 (1990); G. Gantefor, K. H. Meiwes-Broer, and H. O. Lutz, Phys. Rev. A 37, 2716 (1988).

${ }^{9}$ G. F. Ganteför, D. M. Cox, and A. Kaldor, J. Chem. Phys. 96, 4102 (1992); T. N. Kitsopoulos, C. J. Chick, Y. Thao, and D. M. Neumark, ibid. 95, 5479 (1991).

${ }^{10}$ O. Cheshnovsky, S. H. Yang, C. L. Pettiette, M. J. Craycraft, and R. E. Smalley, Rev. Sci. Instrum. 58, 2131 (1987).

"G. Gantefor, H. R. Siekmann, H. O. Lutz, and K. H. Meiwes-Broer, Chem. Phys. Lett. 165, 293 (1990); H. R. Siekmann, Ch. Lüder, J. Faehrmann, H. O. Lutz, and K. H. Meiwes-Broer, Z. Phys. D 20, 417 (1991).

${ }^{12}$ C.-Y. Cha, G. F. Ganteför, and W. Eberhardt (to be published).

${ }^{13}$ G. H. Lee, S. T. Arnold, J. G. Eaton, H. W. Sarkas, K. H. Bowen, C. Ludewig, and H. Haberland, Z. Phys. D 20, 9 (1991).

${ }^{14}$ K. Müller-Dethlefs, M. Sander, and E. W. Schlag, $Z$. Naturforsch. Teil A 39. 1089 (1984); T. N. Kitsopoulos, I. M. Waller, J. G. Loeser, and D. M. Neumark, Chem. Phys. Lett. 159, 300 (1989); G. F. Gantefor, D. M. Cox, and A. Kaldor, J. Chem. Phys. 93, 8395 (1990).

${ }^{15}$ W. C. Wiley and I. H. McLaren, Rev. Sci. Instrum. 26, 1150 (1955).

${ }^{16}$ P. Kruit, F. H. Read, J. Phys. E 16, 313 (1983).

${ }^{17}$ J. Hoe, K. M. Ervin, and W. C. Lineberger, J. Chem. Phys. 93, 6987 (1990).

${ }^{18}$ C. Y. Cha, G. F. Ganteför, and W. Eberhardt (to be published). 